\title{
Multicenter Study of Predictors of Disease Course in Alzheimer Disease (the "Predictors Study”). I. Study Design, Cohort Description, and Intersite Comparisons
}

\author{
*†‡Yaakov Stern, §Marshal Folstein, \|Marilyn Albert, * $\ddagger$ Marcus Richards, \\ ${ }^{*}$ Lisa Miller, §Fred Bylsma, \|Ginette Lafleche, ${ }^{*} \ddagger$ Karen Marder, ${ }^{*}$ Karen Bell, \\ *Mary Sano, †Devangere Devanand, §David Loreck, §Jane Wootten, and \\ ๆJacqueline Bello
}

Departments of *Neurology and $†$ Psychiatry, and the $¥$ Sergievsky Center, Columbia University College of Physicians and Surgeons, New York, New York; \$Department of Psychiatry and Behavioral Sciences, Johns Hopkins University; Baltimore, Maryland; |Departments of Psychiatry and Neurology, Massachusetts General Hospital, Harvard Medical School, Boston, Massachusetts; and IDivision of Neuroradiology, Albert Einstein College of Medicine, New York, New York, U.S.A.

\begin{abstract}
Summary: Clinicians should be able to provide the patient with Alzheimer disease (AD) and the family with an accurate prediction of what to expect, but the variability in the rate of disease progression precludes this. In several previous studies, specific clinical signs such as muscular rigidity, myoclonus, and hallucinations or delusions were associated with rapid progression to a more severe stage of dementia or death. The "Predictors Study," a longitudinal study at three independent sites, was designed to develop a predictor model of the natural history of Alzheimer disease. The study was conducted at three study sites, New York, Baltimore, and Boston in a cohort of 224 patients with early probable AD. This article describes the design and implementation of the Predictors Study, and compares features of the study cohort at baseline across sites. Patients were all at the mild stage of disease at entry and were relatively comparable across sites. Extrapyramidal signs and delusions were common, but myoclonus was rarely observed. Key Words: Multicenter study-Predictors of disease course-Dementia.
\end{abstract}

The rate that Alzheimer disease $(\mathrm{AD})$ progresses is variable (Mayeux et al., 1985; Stern et al., 1987a). We cannot presently predict how long it will be until a patient reaches critical milestones in disease progression such as the inability

Address correspondence and reprint requests to Dr. Y. Stern at 710 West 168 th Street, New York, NY 10032, U.S.A. 
to maintain him or herself without assistance-information that would be useful to both patient and family. The inherent variability in the course of the disease also makes it difficult to evaluate the results of clinical interventions such as drug trials. We initiated a multicenter study to develop reliable methods for estimating the expected length of time until a specific disease outcome occurs, based on information obtained by a clinician early in the course of the disease.

Measurable findings that can help predict disease outcomes have been termed predictors (Wasson et al., 1985). The predictors must be relevant and clearly defined clinical features of the disease, based on easily reproducible observations. Finally, predictors must not be part of the diagnostic criteria for the illness investigated. If, for example, the criteria for an outcome require the presence of a predictor variable, the investigator might conclude that the finding is powerful. This is why we chose to use clinical predictors such as extrapyramidal signs (EPS), as opposed to definitive features of dementia such as memory impairment, as predictors.

An outcome used as the object of prediction must meet several criteria: (a) It must represent a clearly defined event or consequence of the illness that is free of ascertainment bias; (b) it must be relatively easy to establish, based on observations or reports of informants; (c) it must be distinct from diagnostic criteria and from the predictors; (d) it should reflect disease severity and progression in the majority of cases.

Variability of disease progression may reflect phenotypic heterogeneity, phenocopies, or the results of both genetic and environmental factors. Although this is speculative, clear elaboration of predictors of varying disease course would be a first step toward approaching this issue.

This report describes the design and implementation of the "Predictors Study," a multicenter study to identify prediction of decline in $\mathrm{AD}$, and describes baseline data from a cohort recruited across three clinical research sites.

\section{METHODS}

\section{Overview}

Three centers participated in the Predictors Study, Columbia University (CU), Johns Hopkins University School of Medicine $(\mathrm{JH})$, and Massachusetts General Hospital (MGH).

Each center recruited patients who met diagnostic criteria for probable Alzheimer disease (pAD) (McKhann et al., 1984) and were in the early stage of the disease. After determining that inclusion and exclusion criteria were satisfied, historical and demographic information were collected. Neurological, neuropsychological, psychiatric, and functional assessments were performed at the baseline visit and repeated at 6-month intervals. In addition, patients' living situation and medical status were monitored.

The primary predictors chosen for this study were the presence of extrapyramidal signs, myoclonus, and/or psychotic symptoms, based on our previous experience with a smaller cohort of patients with pAD (Mayeux et al., 1985; Stern et al., 1987a; Stern et al., 1990). Additional potential predictive signs, 
based in part on other reports, were presence or absence of history of dementia in first degree relatives, slowed dominant posterior rhythm on EEG, presumed stroke without clinical correlates, and age at onset of symptoms.

Two types of outcomes were utilized: (a) specific disease milestones, such as reaching a specific score on a mental status examination, entry into a nursing home, or death, have dichotomous variables for approaches such as life table analyses, and (b) scores on rating scales of cognitive and functional ability, as descriptors of disease severity for other parametric analyses.

\section{Subjects}

A total of 224 patients (91 from $\mathrm{CU}, 76$ from $\mathrm{JH}$, and 57 from $\mathrm{MGH}$ ) gave informed consent and underwent baseline evaluation.

\section{Methods of Recruitment}

At the CU site, patients were recruited from the Memory Disorders Center and from doctors' private offices through the Alzheimer Disease Research Center. Each consecutive patient who met inclusion and exclusion criteria was enrolled, with the exception of those who would not provide informed consent or who lived too far from the hospital to maintain regular follow-up.

At the $\mathrm{JH}$ site, 47 patients were recruited from the dementia Research Center at the JH Hospital, Department of Psychiatry, 21 were referred from private physicians, seven were recruited from the Department of Neurology at JH Hospital and one was referred from the Psychogeriatric Center at $\mathrm{JH}$ Hospital. All subjects were outpatients.

For the MGH site, 14 patients were recruited from the Hebrew Rehabilitation Center for the Aged in Boston, a chronic long-term care facility, and eight patients were entering a physostigmine study. The remaining patients were recruited through the Geriatric Neurobehavioral Center at $\mathrm{MGH}$, an outpatient service, in the same fashion as at CU.

\section{Inclusion Criteria}

Each patient was required to meet NINCDS-ADRDA criteria for $\mathrm{pAD}$ (McKhann et al., 1984). Intellectual impairment was documented by neuropsychological testing using the standard clinical batteries of each institution. Although actual test batteries differed somewhat at each center, they all included tests of memory, orientation, abstract reasoning, language, attention, and construction.

To ensure that severity of dementia was mild at study entry, all patients were required to have a modified Mini-Mental State Examination (mMMSE) (Mayeux et al., 1981) score of 30 or above (corresponding to approximately 16 on the standard Mini-Mental, Folstein et al., 1975).

Each patient was required to have at least one family member, close friend, or health aid who consented to serve as patient advocate. All patients were required to speak English, either as a primary language or as a fluent second language. Because the present cohort formed the basis of a prospective, longi- 
tudinal study, patients who indicated that they would not return to these centers on a regular basis (e.g. patients who came for a single consultation) were not included.

Patients with "lacunae" or small "vascular" lesions that were clinically and/ or historically silent and were judged to be less than $2 \mathrm{~cm}$ in diameter were included. Diffuse symmetric periventricular lesions, such as those consistent with small-vessel ischemic change or infarcts, or indicative of cerebrospinal fluid (CSF) absorption abnormalities, were not grounds for exclusion. However, patients with cortical lesions of any size or location, or with focal cortical atrophy secondary to an infarct, were excluded.

To ensure accurate initial assessment of psychotic symptoms, all patients were required to be maintained off of antipsychotic medications for at least 1 month before their initial evaluation.

\section{Exclusion Criteria}

We excluded patients with a history or current clinical evidence of schizophrenia or schizoaffective disorder before onset of intellectual decline; any electroconvulsive (ECT) treatment sessions within 2 years of recruitment, or 10 or more ECT treatment sessions at any time; alcohol abuse/dependency [DSMIII-R (1987) criteria] at the time of recruitment; or clinical or historical evidence of stroke.

\section{Procedures}

\section{Data Collected at Initial Evaluation}

Historical and demographic data. Standardized questionnaires were used for age, education, occupation and employment history, family history of dementia or other diseases in first degree relatives, and current medication use. Medical history was also reviewed and coded, with detailed sections for hypertension, diabetes, and myocardial infarction. Specific queries for the presence of thyroid disease, lymphoproliferative disorders, head injury, and Down syndrome were made. The Hachinski Ischemia Scale (Hachinski et al., 1975) was administered to assess risk of vascular disease.

Neurological examination. At the initial visit, a standardized medical and neurological examination was performed. Neurological examinations were repeated on follow-up visits if a change in the patient's medical condition (e.g., hospitalization or new medical diagnosis) had occurred in the intervening period.

Presenting features of dementia. The patient's primary and secondary disease manifestations, as discerned by the examining physician, were recorded, selected from the following list of clinical features: memory change, performance change, language change, disorientation, personality change, depressed mood, behavior change, and psychosis.

Onset dating and features. Based on the interview of the patient and informants, the physician estimated disease duration. Disease onset was also separately 
estimated utilizing a reliable, standardized interview technique (Sano et al., 1987) that asks the informant to date both the onset of clinical features and the latest time they were absent, relative to concrete time markers such as holidays or birthdays.

Brain imaging. All patients underwent a computed tomography (CT) or magnetic resonance imaging (MRI) scan as part of their diagnostic evaluation, and the radiologists' reports were utilized to evaluate the presence of an exclusion criterion (e.g., cortical infarcts).

All available scans were reviewed and rated by a single neuroradiologist at the CU site (JB). Since patients with clinically silent, small subcortical infarcts were included in the study, the presence, frequency, and location of these infarcts were recorded. Ratings of atrophy were made in a format similar to that utilized by the "professional judgment of atrophy" item in the CERAD protocol (Davis et al., 1989), taking the patients' age into account.

Subcortical white matter lesions or infarcts were noted, and rated along the following scale: none, single $(\geq 3 \mathrm{~mm}, \leq 2 \mathrm{~cm})$, few ( $<5$ or $<2$ confluent), multiple ( $\geq 5$ or 2 confluent), diffuse (many confluent). Lesion location was noted and assigned to standardized categories. The presence or absence of frontal or parietal periventricular "caps" (areas of increased signal seen at the poles of the ventricles) was rated in each hemisphere. For MR scans, periventricular "halos" (smooth lines of increased signal at the periventricular margin) were rated as absent, thin (normal), or thick (abnormal).

EEG. Patient EEG reports were extracted in a checklist format. Items included slowed dominant rhythm, focal slowing, intermittent rhythmic slowing $/ \delta$ wave activity, and other slowing and epileptiform activity (focal or generalized).

Psychiatric history. Information concerning past hospitalizations and medication for psychiatric problems was elicited. If a history of depression was identified, the kind of treatment, if any, was recorded.

Family history of dementia. A list of first-degree relatives of each patient was compiled, along with age at, and cause of, death. Also recorded was whether each family member has or had dementia, based on the report of the informants.

\section{Data Collected at Initial and Follow-up Visits}

The following assessments were completed at the initial and at all subsequent follow-up evaluations.

Medication. Since specific medications might influence evaluated performance, current medications at the time of assessment, along with their indication and efficacy, were recorded on a standardized checklist form. Amount and form of alcohol consumed was also recorded.

Seizures. The presence and frequency of seizures, whether or not suggested by EEG abnormality, were recorded.

Any repeat imaging or EEG studies were noted and their results recorded.

Medical and neurological examinations were repeated if any significant medical event occurred in the 6 months before a visit. 


\section{Assessment of Potential Predictive Signs}

The following assessments occurred at the initial and at each subsequent 6month visit.

Extrapyramidal signs rating. Selected items from the Unified Parkinson's Disease Rating Scale (Stern, 1983) were used, comprising ratings of voice, facial immobility, resting tremor, rigidity (neck and each limb), brady/hypokinesia, posture and gait [see Richards et al. (1991) for complete form]. Severity of each sign was rated as either absent $(0)$, slight $(1)$, mild-moderate (2), marked (3), or severe (4). Signs were coded as either idiopathic, induced by current neuroleptic medication, or possibly induced by previous neuroleptic medication. The presence of chorea, myoclonus, and other dyskinesias was also recorded. Interrater reliability of this examination has been established (Richards et al., 1991).

Psychosis and behavioral changes. A semistructured interview, the Columbia University Scale for Psychopathology in Alzheimer's Disease (CUSPAD) (Devenand et al., 1992) elicited information about symptoms of delusions, hallucinations, illusions, depressed mood, and other specific behavioral signs occurring during the month before assessment. Specific delusions queried included paranoid delusions, delusions of abandonment, somatic delusions, and misidentifications. The CUSPAD assessed delusion severity based on frequency and the readiness of the patient to accept the truth if corrected. Only if the patient was resistant to accepting correction was a delusion considered to meet DSM-III-R (1987) diagnostic criteria. Hallucinations and illusions, if present, were rated as either vague or clear. Behavioral problems, including wandering, verbal outbursts, physical threats and/or violence, agitation or restlessness, and nighttime confusion were rated as present or absent. The presence of depressed mood was scored on a 5-point severity scale.

Depression. In order to supplement the CUSPAD rating of depressive symptoms, the Hamilton Depression Scale (Hamilton, 1960) was administered jointly (where possible) to the patient and the informant. An interview format was used to standardize administration (Williams, 1988).

\section{Assessment of Disease Outcomes}

Cognitive assessment. Intellectual function was examined using the mMMSE (Mayeux et al., 1981). This is based on the standard Mini-Mental State Examination (Folstein et al., 1975) and includes the Wechsler Adult Intelligence Scale Digit Span subtest (Wechsler, 1955), and additional attention/calculation, general knowledge, language, and construction items. The maximum score on this test is 57. This is a valid and reliable instrument (Stern et al., 1987b) that is brief yet informative. Formal instructions and guides for its use have been developed. The mMMS yields subscores that are descriptive of specific intellectual functions: orientation, registration, recall, digit span, attention, naming, language, and construction. We consider an mMMS score of 15 , representative of moderate to severe $\mathrm{pAD}$, as a specific end-point for prediction. 
After mMMSE administration, the examiner noted whether the patient was mute or uncooperative, since this can affect interpretation of the test. At baseline testing, no patient was rated in these categories.

Functional assessment. Functional capacity was rated using the Blessed Dementia Rating Scale (Part 1) (BDRS) (Blessed et al., 1968). The reliability and validity of this instrument have been established and it has proven to be useful in evaluating longitudinal changes in intellectual function. We have developed a structured interview to guide and standardize BDRS administration. We have also developed subscores for the BDRS based on factor analysis (Stern et al., 1990). These factors are cognitive, personality, apathy, and basic self-care.

We consider a BDRS score of 10 as a specific end-point for prediction. We have also utilized specific scores as outcomes for the individual factors (Stern et al., 1990).

Dependence rating. This scale (Stern et al., 1992) was adapted by investigators at Columbia from the work of Gurland et al. (1978), who assessed dependence in community dwelling elders. Since a patient at home might receive care comparable to that at a skilled nursing facility, the intent of the scale was to document the extent of the patient's dependence, or need for the supervision and care of others. Part 1 of this scale assesses the amount of assistance required by patients. Items range from low level care such as supervision when outdoors to those indicative of a high level of dependence such as nasogastric feeding. A dependence level was derived from these items, ranging from 0 , totally independent, to 5, totally dependent. Part 2 recorded the rater's impression of the level of institutional or equivalent care received by the patient, irrespective of the patient's actual location. Categories included limited home care, adult home (a supervised setting with regular assistance in most activities), and health-related facility.

Outcomes for the dependence scale include reaching a specific dependence stage and reaching a level of care equivalent to a health-related facility.

Current status. At each 6-month evaluation, indicators of the patient's current status are recorded. These include work status, living situation (e.g., at home, in nursing home), or death (and cause of death).

The Clinical Dementia Rating scale (CDR) (Hughes et al., 1982) was used to evaluate the patient at each measurement point to provide a global assessment of the severity of dementia. Dementia severity was rated mild (1), moderate (2), severe (3), profound (4), or terminal (5). This rating is based on all data collected and an interview with the patient and informant.

\section{Data Base Entry and Validation}

A data base was established at CU using SIR-DBMS software [SIR (USA), Deerfield, IL, U.S.A.] implemented on a network of microcomputers. Data was sent from all three sites to the study coordinator, who checked it for consistency and completeness before entry. 


\section{Study Personnel and Training}

Medical/neurological examination. All initial and follow-up neurological/ medical examinations were conducted by physicians (neurologists or psychiatrists). All other interviews were conducted by staff trained at each site. All evaluations were supervised by the principal investigator at that site.

To ensure standardization of data collection methods, a meeting of the personnel of the three study sites was held before initiating the study to review all forms and procedures. Five patients and their informants (not in the current study) served as practice subjects.

Following this initial training session, visits of $\mathrm{JH}$ and $\mathrm{MGH}$ investigators to the CU site, as well as CU investigators to the other sites were arranged. Particular care was taken with training and monitoring of the rating of extrapyramidal signs, since this relies heavily on clinical judgment. A total of five additional training/review sessions were conducted at the three sites. In each case, a series of patients were jointly rated. CU investigators served as the criterion raters, and differences in ratings between investigators were discussed and resolved.

For the final two sessions, formal reliability trials were held, with one rater from each of the three sites independently rating the same 14 patients. Agreement for the presence or absence of EPS across the three raters was $100 \%$. Consistency of the EPS ratings across raters, estimated with intraclass correlation (ICC), was excellent ( $\mathrm{ICC}=0.87)$.

Similar additional sessions were held for other aspects of the study protocol, with emphasis on assessments that relied on clinical judgment to any degree. These included CUSPAD, onset rating, and the Hamilton Depression Scale.

In addition to the formal sessions that were designed to enhance and monitor intersite reliability, the CU site served as a clearing house for clarification of emergent issues in the application of the study protocol. Finally, data from all three sites was closely monitored for deviation from protocol. This included evaluation of written notes accompanying the ratings of most items on the assessment forms.

\section{Statistical Analysis}

The primary analyses compared clinical and performance measures across the three study sites. $\chi^{2}$ analyses were utilized for frequency data and analyses of variance or covariance for continuous variables. In all cases below, an $\alpha$ value of 0.05 or less was required for noting between group differences.

\section{RESULTS}

\section{Demographic Features}

Two hundred twenty-four patients were recruited into the study. Approximately 345 additional patients with $A D$ were screened but did not meet study entry criteria or refused to participate (120 at $\mathrm{CU}, 115$ at $\mathrm{JH}$, and 110 at $\mathrm{MGH}$ ). Means and/or breakdowns for age, education, ethnic group, living situation, 
marital status, and employment status are shown in Table 1 , for the entire study and for each site separately.

The proportion of men and women was evenly distributed across the study sites. There was no statistical difference in the educational level of the patients between the sties. However, a significant age difference across sites was found: Mean age of patients from MGH was notably higher than that for the other two sites. Almost all patients were white and were born in the United States. English was the predominant language spoken at home, although a small number of patients from the CU site spoke Spanish or another language at home.

Seventeen patients were reported to be working full or part time at the time of the initial assessment; 26 were reported to have worked within the previous 6 months. Approximately half of the patients with an employment history had retired under normal circumstances; $21 \%$ stopped work early as a result of dementia.

\section{Medical and Psychiatric History}

Approximately $30 \%$ of patients reported a history of hypertension and over $20 \%$ of patients a history of hypercholesteremia (Table 2). A disproportionate number of these were from the $\mathrm{JH}$ site, as were patients with hypertriglyceridemia. Cardiac disease was defined as having at least one of the following: myocardial infarction, congestive heart failure, valvular heart disease, angina, atrial fibrillation or other cardiac arrhythmias. According to this definition, 56 patients had at least one sign of cardiac disease; there was no significant difference in frequency across the sites.

No other aspect of medical history differed significantly across sites. Fifty (23\%) of the patients had been treated for depression at some time in the past; seven had had ECT, 42 had received medication for depression, and 19 underwent therapy or counseling.

\section{Medical and Neurological Evaluation}

\section{Presenting Complaints}

The most frequent presenting complaint was a memory deficit $(81 \%)$, followed by difficulty with performing specific tasks. The distribution of presenting signs was comparable across sites (Table 3 ).

\section{Medical Examination}

Fifty-eight patients $(28 \%)$ had at least one abnormal finding on medical evaluation; none was felt to contribute to the presentation of dementia. Fifty-eight patients were being treated for hypertension and eight met criteria for hypertension based on a blood pressure greater than 160/95. Frequency of treated hypertension or hypertension at the time of examination did not differ across sites.

Mean Hachinski score across all sites was $1.52(\mathrm{SD}=1.35)$; scores did not differ significantly across sites. 
TABLE 1. Demographic features of the $p A D$ cohort

\begin{tabular}{|c|c|c|c|c|}
\hline & Overall & $\mathrm{CU}$ & JH & MGH \\
\hline$n$ & 224 & 91 & 76 & 57 \\
\hline \multicolumn{5}{|l|}{ Sex } \\
\hline $\mathbf{M}$ & $91(40.6 \%)$ & $40(44.0 \%)$ & $31(40.8 \%)$ & $20(35.1 \%)$ \\
\hline F & $133(59.4 \%)$ & $51(56.0 \%)$ & $45(59.2 \%)$ & $37(64.9 \%)$ \\
\hline Age (yr) & $73.09(8.74)$ & $71.02(8.04)$ & $72.26(7.82)$ & $77.51(9.52)$ \\
\hline Education (yr) & $13.12(3.65)$ & $13.36(3.62)$ & $12.42(4.14)$ & $13.67(2.81)$ \\
\hline \multicolumn{5}{|l|}{ Race/ethnicity: } \\
\hline White & $208(92.9 \%)$ & $83(91.2 \%)$ & $68(89.5 \%)$ & $57(100 \%)$ \\
\hline Black & $15(6.7 \%)$ & $7(7.7 \%)$ & $8(10.5 \%)$ & 0 \\
\hline Hispanic & $8(3.4 \%)$ & $6(6.6 \%)$ & $2(2.6 \%)$ & 0 \\
\hline Born in U.S.A. & $193(86.5 \%)$ & $71(78.0 \%)$ & $71(94.7 \%)$ & $51(89.5 \%)$ \\
\hline Speak English at home & $217(96.9 \%)$ & $84(92.3 \%)$ & $76(100 \%)$ & $57(100 \%)$ \\
\hline \multicolumn{5}{|l|}{ Marital status } \\
\hline Married & $142(63.4 \%)$ & $65(71.4 \%)$ & $45(59.2 \%)$ & $32(56.1 \%)$ \\
\hline Widowed & $66(29.5 \%)$ & $18(19.8 \%)$ & $25(32.9 \%)$ & $23(40.4 \%)$ \\
\hline Divorced/separated & $14(6.2 \%)$ & $6(6.6 \%)$ & $6(7.9 \%)$ & $2(3.6 \%)$ \\
\hline \multicolumn{5}{|l|}{ Work status: } \\
\hline Working full or $\mathrm{P} / \mathrm{T}$ & $17(7.6 \%)$ & $10(11.0 \%)$ & $3(3.9 \%)$ & $4(7.0 \%)$ \\
\hline Normal retirement & $109(48.7 \%)$ & $35(38.5 \%)$ & $43(56.6 \%)$ & $31(54.4 \%)$ \\
\hline Retired due to $\mathrm{pAD}$ & $48(21.4 \%)$ & $28(30.8 \%)$ & $14(18.4 \%)$ & $6(10.5 \%)$ \\
\hline Homemaker & $24(10.7 \%)$ & $9(9.9 \%)$ & $4(5.3 \%)$ & $11(19.3 \%)$ \\
\hline
\end{tabular}

In this and all following tables, values in parentheses represent either percent or standard deviation.

\section{Neurological Examination}

Most features of the neurological examination were comparable across sites (Table 4). However, distributions differed significantly across sites for minor speech abnormalities and reflexes. In both cases, higher frequencies of abnormalities were noted in the $\mathrm{JH}$ group and probably represent differential sensitivity in endorsing these items.

\section{Medications}

Medications taken by patients at the time of recruitment are shown in Table 5. In line with inclusion criteria, no patients were taking antipsychotic medications. Patients taking thyroid medications were not currently hypothyroid. Twenty patients were active participants in research drug trials for the treatment of $A D$; their drug/placebo status could not be determined at entry. Drug protocols include oral physostigmine, piracetam, acetyl-l-carnitine and tetrahydroaminoacridine.

\section{Laboratory Studies}

\section{Diagnostic Imaging Studies}

All patients underwent either a CT or MRI study as part of their diagnostic workup; 150 had CT, 90 had MRI, and 16 had both.

One hundred fifty-eight scans (55 MRI, $103 \mathrm{CT}$ ) were reviewed at the CU site by a single neuroradiologist (JB). Approximately $89 \%$ of the scans were 
TABLE 2. Features of medical and psychiatric history in the cohort

\begin{tabular}{|c|c|c|c|c|}
\hline & Overall & $\mathrm{CU}$ & ЈH & MGH \\
\hline \multicolumn{5}{|l|}{ Medical History } \\
\hline Hypertension & $66(29.7 \%)$ & $25(27.8 \%)$ & $28(37.3 \%)$ & $13(22.8 \%)$ \\
\hline Diabetes & $13(5.9 \%)$ & $4(4.4 \%)$ & $6(8.1 \%)$ & $3(5.5 \%)$ \\
\hline Cardiac risk factor & $56(25.5 \%)$ & $20(22.5 \%)$ & $20(27.0 \%)$ & $16(28.1 \%)$ \\
\hline Hypercholesterolemia & $45(20.7 \%)$ & $15(17.6 \%)$ & $28(37.3 \%)$ & $2(3.5 \%)$ \\
\hline Hypertriglyceridemia & $10(4.7 \%)$ & $3(3.6 \%)$ & $7(9.5 \%)$ & 0 \\
\hline Epilepsy/seizures & $4(1.8 \%)$ & 0 & $3(4.0 \%)$ & $1(1.8 \%)$ \\
\hline Head trauma with LOC & $5(2.3 \%)$ & $1(1.1 \%)$ & $2(2.7 \%)$ & $2(3.5 \%)$ \\
\hline Autoimmune disease & $8(3.6 \%)$ & $5(5.6 \%)$ & $1(1.3 \%)$ & $2(3.5 \%)$ \\
\hline Chronic alcohol abuse & $11(5.0 \%)$ & $6(6.7 \%)$ & $5 \quad(6.7 \%)$ & 0 \\
\hline Drug abuse & $2(0.9 \%)$ & 0 & $2(2.7 \%)$ & 0 \\
\hline \multicolumn{5}{|l|}{ Psychiatric history } \\
\hline Treated for depression & $50(22.6 \%)$ & $21(23.6 \%)$ & $17(22.7 \%)$ & $12(21.1 \%)$ \\
\hline ECT & $7(3.2 \%)$ & $5(5.6 \%)$ & $1(1.3 \%)$ & $1(1.8 \%)$ \\
\hline Drug treatment & $42(19.0 \%)$ & $16(18.0 \%)$ & $16(21.3 \%)$ & $10(17.5 \%)$ \\
\hline Therapy/counseling & $19(8.7 \%)$ & $8(9.1 \%)$ & $6(8.0 \%)$ & $5(8.9 \%)$ \\
\hline
\end{tabular}

LOC, loss of consciousness; ECT, electroconvulsive treatment.

rated as reflecting at least mild atrophy. More notable atrophy was noted in patients from the CU study population (Table 6).

At least one white matter lesion or lacune was noted in $55 \%$ of the patients. The distribution of lesions was similar across study sites. The distribution of frontal caps was similar across sites, but there was a significant difference in the frequency of parietal caps. The distribution of halos was also comparable across sites.

\section{EEG Studies}

One hundred thirty-six patients (65.7\%) had an EEG study as part of their diagnostic evaluation. EEG findings for these patients are shown in Table 7. The distribution of slowing was comparable across sites.

\section{Predictive Signs}

\section{EPS}

Across all three sites, five patients had extrapyramidal signs that were judged to be definitely related to current medications; in nine others, EPS might have resulted in part from medication use. These 14 patients were excluded from analysis of EPS.

Overall, $40.2 \%$ of the study patients had at least one EPS sign rated as at least slight. The distribution of these patients varied significantly across sites, with more EPS noted at $\mathrm{JH}(52.6 \%)$ and $\mathrm{MGH}(50.9 \%)$ than at $\mathrm{CU}(23.1 \%)$. Using a more restrictive criterion for the presence of EPS requiring at least one sign rated at the mild-moderate range, $15.2 \%$ of patients overall had EPS. The distribution across sites was still uneven, with more EPS at MGH (24.6\%) and $\mathrm{JH}$ $(18.4 \%)$ than at $\mathrm{CU}(6.6 \%)$. Figure 1 summarizes the percentage of patients at 
TABLE 3. Primary presenting complaint of patient and/or informant to the examining neurologist

\begin{tabular}{|c|c|c|c|c|}
\hline & Overall & $\mathrm{CU}$ & $\mathrm{JH}$ & MGH \\
\hline Memory deficit & $179(81.0 \%)$ & $75(83.3 \%)$ & $56(75.7 \%)$ & $48(84.2 \%)$ \\
\hline Performance problem & $14(6.3 \%)$ & $6(6.7 \%)$ & $4(5.4 \%)$ & $4(7.0 \%)$ \\
\hline Language problem & $8(3.6 \%)$ & $4(4.4 \%)$ & $3(4.1 \%)$ & $1(1.8 \%)$ \\
\hline Disorientation & $8(3.6 \%)$ & 0 & $6(8.1 \%)$ & $2(3.5 \%)$ \\
\hline Personality change & $5 \quad(2.3 \%)$ & $2(2.2 \%)$ & $1(1.4 \%)$ & $(3.5 \%)$ \\
\hline Depressed mood & $3(1.4 \%)$ & $1(1.1 \%)$ & $2(2.7 \%)$ & 0 \\
\hline Behavior change & $3(1.4 \%)$ & $2(2.2 \%)$ & $1(1.4 \%)$ & 0 \\
\hline Psychosis & $1(0.5 \%)$ & 0 & $1(1.4 \%)$ & 0 \\
\hline
\end{tabular}

each site who were rated as either slight or mild-moderate for each EPS. Again, frequencies for individual signs differed across site for speech, masked facies, rigidity, posture, bradykinesia, and gait.

Patients with EPS were older than those without EPS (mean age 75.4 and 70.3, respectively). Duration of illness was comparable in the two groups, however. EPS were noted more often in older patients, and this accounts to some degree for different frequencies across sites. However, differences in frequency of EPS across sites were still noted in analyses that controlled for age.

The frequency of EPS in the pAD patients in this study was higher than that seen in an age-matched group of healthy elderly individuals (Bell et al., 1992).

\section{Myoclonus}

Only three patients in the entire study cohort had myoclonus, two at $\mathrm{JH}$ and one at MGH. In all cases myoclonus occurred only during sleep. Two patients at $\mathrm{JH}$ were also diagnosed with tardive dyskinesia.

\section{Psychotic and Behavioral Symptoms}

Overall, $24.3 \%$ of the patients met criteria for having had a delusion in the past month. Most common were paranoid delusions. The frequency of delusions was comparable across study sites. The frequency of hallucinations and illusions

TABLE 4. Frequency of abnormalities noted on the neurological examination

\begin{tabular}{lrcrcr}
\hline & Overall & CU & JH & MGH \\
\hline Consciousness & 0 & 0 & 0 & 0 \\
Speech/language & $21(9.5 \%)$ & $8(8.8 \%)$ & $13(17.6 \%)$ & 0 \\
Visual fields & $4(1.8 \%)$ & $2(2.2 \%)$ & $2(2.7 \%)$ & 0 \\
Eye movements & $5(2.3 \%)$ & $5(5.6 \%)$ & 0 & 0 \\
Other cranial nerves & $9(4.1 \%)$ & $3(3.3 \%)$ & $5(6.7 \%)$ & $1(1.8 \%)$ \\
Motor & $9(4.1 \%)$ & $4(4.4 \%)$ & $3(4.0 \%)$ & $2(3.5 \%)$ \\
Sensory & $10(4.5 \%)$ & $7(7.8 \%)$ & $2(2.7 \%)$ & $1(1.8 \%)$ \\
Cerebellar & $9(4.1 \%)$ & $6(6.7 \%)$ & $3(4.0 \%)$ & 0 \\
Movements & $5(2.3 \%)$ & $4(4.4 \%)$ & $1(1.3 \%)$ & 0 \\
Reflexes & $32(14.4 \%)$ & $12(13.3 \%)$ & $17(22.7 \%)$ & $3(5.3 \%)$ \\
\hline
\end{tabular}


TABLE 5. Summary of standard and experimental medications currently prescribed

\begin{tabular}{lcccc}
\hline & Combined & CU & JH & MGH \\
\hline Antidepressant & $22(9.6 \%)$ & $8(8.8 \%)$ & $9(11.8 \%)$ & $5(9.0 \%)$ \\
Sedative/hypnotics & $12(5.3 \%)$ & $8(8.8 \%)$ & $3(3.9 \%)$ & $1(1.8 \%)$ \\
Thyroid medication & $7(3.1 \%)$ & $1(1.1 \%)$ & $1(1.3 \%)$ & $5(8.8 \%)$ \\
Research medications & $20(8.9 \%)$ & $7(7.7 \%)$ & $8(10.5 \%)$ & $2(3.6 \%)$ \\
\hline
\end{tabular}

is summarized in Table 8. These were less frequent than delusions and their distribution also did not differ significantly across study sites.

Other behavioral problems were frequent, occurring in $52.3 \%$ of the cohort. The frequency of these problems differed significantly across the three sites.

Based on the CUSPAD, depressed mood (minimal to severe in intensity) was reported in $42.3 \%$ of the patients. There was a significant difference in severity of depressive symptoms across sites, with greater severity noted at CU. Similarly, Hamilton Depression Scale scores differed significantly across sites, with higher scores noted at $\mathrm{CU}$.

\section{Disease Severity Descriptors}

\section{General Severity Descriptors}

A review of general disease severity descriptors (Table 9) indicates that the cohort was early in the course of pAD and only mildly demented.

Mean duration of illness, as estimated by the examining physician, was 4 years, and did not differ significantly across sites. As estimated by the onset interview, mean duration was 4.26 years, slightly higher, and did not differ

TABLE 6. Standardized ratings of features of CT and MRI scans by a single neuroradiologist

\begin{tabular}{|c|c|c|c|c|}
\hline & Combined & $\mathrm{CU}$ & $\mathrm{JH}$ & MGH \\
\hline MRI $(n)$ & 55 & 26 & 28 & 1 \\
\hline CT $(n)$ & 103 & 32 & 27 & 44 \\
\hline \multicolumn{5}{|l|}{ Atrophy } \\
\hline none & $17(10.8 \%)$ & 0 & $9(16.4 \%)$ & $8(17.8 \%)$ \\
\hline mild & $115(72.8 \%)$ & $43(74.1 \%)$ & $37(67.3 \%)$ & $35(77.8 \%)$ \\
\hline moderate & $25(15.8 \%)$ & $14(24.1 \%)$ & $9(16.4 \%)$ & $2(4.4 \%)$ \\
\hline marked & $1(0.6 \%)$ & $1(1.7 \%)$ & 0 & 0 \\
\hline \multicolumn{5}{|l|}{ Lesions } \\
\hline none & $70(44.3 \%)$ & $27(46.6 \%)$ & $25(45.5 \%)$ & $18(40.0 \%)$ \\
\hline single & $34(21.5 \%)$ & $12(20.7 \%)$ & $12(21.8 \%)$ & $10(22.2 \%)$ \\
\hline few & $35(22.2 \%)$ & $11(19.0 \%)$ & $9(16.4 \%)$ & $15(33.3 \%)$ \\
\hline multiple & $18(11.4 \%)$ & $8(13.8 \%)$ & $8(14.5 \%)$ & $2(4.4 \%)$ \\
\hline diffuse & $1(0.6 \%)$ & 0 & $1(1.8 \%)$ & 0 \\
\hline \multicolumn{5}{|l|}{ Caps } \\
\hline frontal & $42(26.6 \%)$ & $11(19.0 \%)$ & $19(34.5 \%)$ & $12(26.7 \%)$ \\
\hline parietal & $55(34.8 \%)$ & $20(34.4 \%)$ & $25(45.4 \%)$ & $10(22.2 \%)$ \\
\hline Halo (in MR only) & $22(38.6 \%)$ & $8(30.7 \%)$ & $13(44.8 \%)$ & $1(50.0 \%)$ \\
\hline
\end{tabular}


TABLE 7. Features of EEG

\begin{tabular}{lrrrr}
\hline & \multicolumn{1}{c}{ Combined } & CU & JH & \multicolumn{1}{c}{ MGH } \\
\hline Had EEG & $140(62.8 \%)$ & $64(70.3 \%)$ & $52(69.3 \%)$ & $24(42.1 \%)$ \\
Slow dominant thythm & $45(32.4 \%)$ & $24(37.5 \%)$ & $14(27.5 \%)$ & $7(29.2 \%)$ \\
Focal slowing & $18(13.0 \%)$ & $8(12.7 \%)$ & $10(19.6 \%)$ & 0 \\
Intermittent rhythmic & $8(5.8 \%)$ & $3(4.7 \%)$ & $5(10.0 \%)$ & 0 \\
$\quad$ slowing & $3(2.2 \%)$ & $1(1.6 \%)$ & $2(4.0 \%)$ & 0 \\
Focal epileptiform & $3(1.3 \%)$ & 0 & $2(2.7 \%)$ & $1(1.8 \%)$ \\
Seizure & & & & \\
\hline
\end{tabular}

significantly across sites $(p<0.07)$. Mean CDR was 1.1 , reflecting the fact that the great majority of patients $(89.7 \%$ ) were rated as having a CDR of 1 , mild dementia. The remainder were rated as CDR 2. Distribution of CDR ratings did not differ significantly across sites.
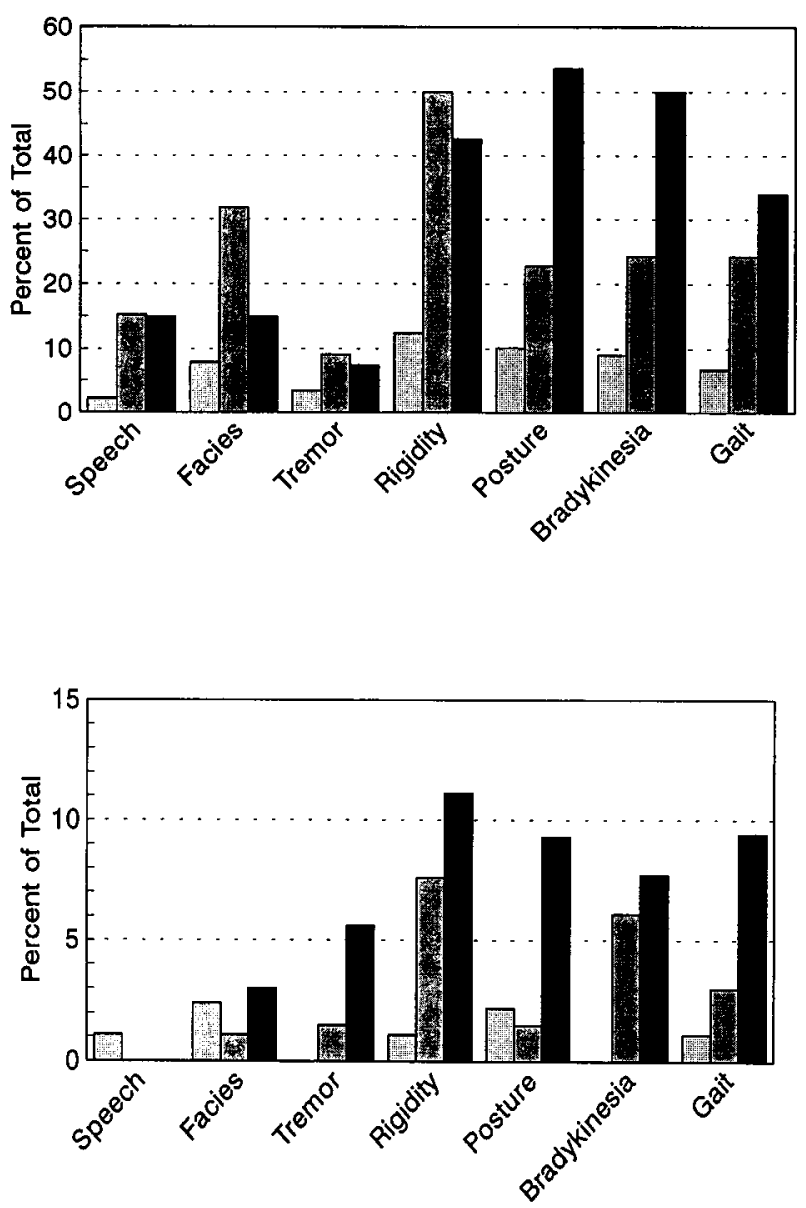

FIG. 1. Percentage of patients at each study site rated as having specific extrapyramidal signs. Top: Signs rated at "slight" severity or higher. Bottom: Signs rated at "mild-moderate" severity or higher. Hatched bars represent CU patients; gray bars represent JH patients; black bars represent MGH patients. 
TABLE 8. Current psychiatric status as assessed by the CUSPAD and the Hamilton Depression Scale

\begin{tabular}{lccccc}
\hline & Combined & \multicolumn{1}{c}{ CU } & JH & MGH \\
\hline Any delusion & $54(24.3 \%)$ & $22(24.4 \%)$ & $20(26.7 \%)$ & $12(21.1 \%)$ \\
$\quad$ Paranoid delusion & $32(14.4 \%)$ & $14(15.6 \%)$ & $11(14.7 \%)$ & $7(12.3 \%)$ \\
Delusion of abandonment & $3(1.4 \%)$ & 1 & $(1.1 \%)$ & 0 & $2(3.5 \%)$ \\
$\quad$ Somatic delusion & $3(1.4 \%)$ & $2(2.2 \%)$ & 0 & $1(1.8 \%)$ \\
Misidentification & 0 & & & & \\
Other delusion & $4(1.8 \%)$ & 0 & $3(4.0 \%)$ & $1(1.8 \%)$ \\
Hallucinations & $7(3.2 \%)$ & $2(2.2 \%)$ & $4(5.3 \%)$ & $1(1.8 \%)$ \\
$\quad$ Vague & $11(5.0 \%)$ & $3(3.3 \%)$ & $3(4.0 \%)$ & $5(8.8 \%)$ \\
$\quad$ Clear & $7(3.2 \%)$ & $2(2.2 \%)$ & $4(5.3 \%)$ & $1(1.8 \%)$ \\
Illusions & $116(52.3 \%)$ & $55(61.1 \%)$ & $40(53.3 \%)$ & $21(36.8 \%)$ \\
Behavioral problems & $94(42.3 \%)$ & $43(47.8 \%)$ & $29(38.7 \%)$ & $22(38.6 \%)$ \\
Depressed mood & $3.22(4.06)$ & $5.26(4.80)$ & $2.68(3.56)$ & $1.89(3.04)$ \\
Hamilton Depression Scale & & & & & \\
\hline
\end{tabular}

Another approach to disease severity is to assess patients' living situation. Only 15 patients $(6.7 \%)$ were living in a nursing home. This reflects recruitment at MGH, where 14 patients were recruited from a chronic long-term care facility. Of those not living in a clinical facility, the majority (64.4\%) were living at home, with their spouse, partner, children, family, or friends. Notably, 33 (17.3\%) were living alone at the time of first assessment.

Even if patients are living at home, they may receive the equivalent of nursing home care. The "equivalent" care is assessed by Part 2 of the dependence scale. Based on interviewers' impressions, 55.6\% of the patients had the equivalence of limited home care whereas $35.9 \%$ received the equivalent of custodial care. Only 19 , including the 15 actually in a nursing home, were judged to be receiving the equivalent of care in a skilled nursing facility.

TABLE 9. Indicators of severity of Alzheimer disease

\begin{tabular}{|c|c|c|c|c|}
\hline & Combined & $\mathrm{CU}$ & JH & MGH \\
\hline \multicolumn{5}{|l|}{ CDR } \\
\hline Mean & $1.10(0.30)$ & $1.11(0.31)$ & $1.12(0.32)$ & $1.07(0.26)$ \\
\hline Mild $(C D R=1)$ & $201(89.7 \%)$ & $81(89.0 \%)$ & $67(88.2 \%)$ & $53(93.0 \%)$ \\
\hline Moderate $(C D R=2)$ & $23(10.3 \%)$ & $10(11.0 \%)$ & $9(11.8 \%)$ & $4(7.0 \%)$ \\
\hline \multicolumn{5}{|l|}{ Mean estimated duration } \\
\hline Neurologist & $3.98(2.60)$ & $3.36(1.84)$ & $4.72(3.09)$ & $4.00(2.72)$ \\
\hline Onset interview & $4.26(3.47)$ & $3.62(2.58)$ & $4.75(4.16)$ & $4.67(3.64)$ \\
\hline \multicolumn{5}{|l|}{ Living status } \\
\hline Alone & $35(16.8 \%)$ & $17(18.7 \%)$ & $12(16.0 \%)$ & $6(14.3 \%)$ \\
\hline With spouse at home & $133(63.9 \%)$ & $56(61.5 \%)$ & $44(58.7 \%)$ & $33(78.6 \%)$ \\
\hline With family at home & $204(91.1 \%)$ & $91(100.0 \%)$ & $72(94.7 \%)$ & $41(71.9 \%)$ \\
\hline Retirement home & $3(1.3 \%)$ & 0 & $1(1.3 \%)$ & $2(3.5 \%)$ \\
\hline Nursing home & $15(6.7 \%)$ & 0 & $1(1.3 \%)$ & $14(24.6 \%)$ \\
\hline \multicolumn{5}{|l|}{ Estimated equivalent care } \\
\hline Limited home care & $124(55.6 \%)$ & $(41.8 \%)$ & $53(70.7 \%)$ & $33(57.9 \%)$ \\
\hline Custodial care & $80(35.9 \%)$ & $(52.7 \%)$ & $22(29.3 \%)$ & $10(17.5 \%)$ \\
\hline Skilled nursing facility & $19(8.5 \%)$ & $(5.5 \%)$ & 0 & $14(24.6 \%)$ \\
\hline
\end{tabular}


TABLE 10. Mean scores on the modified Mini-Mental State Examination and its component subtests

\begin{tabular}{lcrrrr}
\hline & $\begin{array}{c}\text { Maximum } \\
\text { score }\end{array}$ & \multicolumn{1}{c}{ Combined } & CU & JH & \multicolumn{1}{c}{ MGH } \\
\hline Total & 57 & $37.74(5.55)$ & $37.69(5.20)$ & $36.82(5.40)$ & $39.05(6.1)$ \\
Orientation & 10 & $6.19(2.21)$ & $6.21(2.20)$ & $6.14(2.06)$ & $6.23(2.4)$ \\
Registration & 3 & $2.82(0.52)$ & $2.78(0.57)$ & $2.79(0.57)$ & $2.93(0.3)$ \\
Digit span total & 10 & $9.46(1.87)$ & $9.24(1.65)$ & $9.50(2.18)$ & $9.75(1.7)$ \\
$\quad$ Forward & 6 & $5.80(1.10)$ & $5.66(1.04)$ & $5.95(1.23)$ & $5.82(1.0)$ \\
$\quad$ Backward & 4 & $3.69(0.98)$ & $3.58(0.97)$ & $3.63(0.99)$ & $3.93(0.9)$ \\
Attention/calculation & 7 & $3.29(2.42)$ & $3.09(2.45)$ & $3.03(2.42)$ & $3.95(2.2)$ \\
Presidents & 5 & $1.46(1.28)$ & $1.81(1.41)$ & $1.25(1.16)$ & $1.18(1.0)$ \\
Recall & 3 & $0.45(0.74)$ & $0.34(0.70)$ & $0.34(0.62)$ & $0.75(0.8)$ \\
Naming & 10 & $7.77(1.93)$ & $7.84(2.08)$ & $7.87(1.61)$ & $7.53(2.0)$ \\
Language & 7 & $5.94(1.10)$ & $6.04(1.05)$ & $5.66(1.09)$ & $6.14(1.1)$ \\
Construction & 2 & $0.88(0.78)$ & $0.69(0.76)$ & $0.95(0.80)$ & $1.10(0.7)$ \\
\hline
\end{tabular}

\section{Modified Mini-Mental State Examination}

Mean mMMSE score, along with means for each subscore are shown in Table 10. Since no patients with a mMMSE score below 30 were entered into the study, the distribution of the total score was significantly positively skewed $(z$ $=1.44, p=0.032$ ). These scores did not differ significantly across study sites.

\section{Blessed Dementia Rating Scale and Dependence Scale}

Mean total score for the Blessed Dementia Rating Scale (Part I) was 8.08. Overall, Blessed scores were higher at the CU site (Table 11). Item scores were also subdivided based on a factor analysis described previously. Scores on the cognitive factor, comprising the first seven test items suggested some difficulty with orientation, calculation, and chores. In contrast, scores were generally low (indicating preserved function) for the Basic Self-Care factor, which assesses eating, dressing, and toileting, again indicative of relatively mild dementia. Scores for the two personality change factors indicate the presence of both positive and negative personality changes.

The mean dependence level indicates the need for help on only the earlier items on the dependence scale, such as requiring reminder to manage chores, or

TABLE 11. Functional status and dependence, rated with the Blessed Dementia Rating Scale (Part 1) and the Dependence Scale

\begin{tabular}{lcccc}
\hline & Combined & CU & JH & MGH \\
\hline Mean BDRS score & $8.08(3.47)$ & $8.62(3.58)$ & $7.76(3.62)$ & $7.62(3.00)$ \\
BDRS factor scores & & & & \\
Cognitive & $3.03(1.32)$ & $2.81(1.16)$ & $3.19(1.28)$ & $3.16(1.56)$ \\
Personality & $2.04(1.64)$ & $2.44(1.62)$ & $1.78(1.62)$ & $1.75(1.61)$ \\
Apathy & $1.74(0.97)$ & $1.89(0.96)$ & $1.50(1.05)$ & $1.82(0.83)$ \\
Basic Self Care & $0.53(0.90)$ & $0.64(1.04)$ & $0.51(0.85)$ & $0.37(0.67)$ \\
Dependence level & $2.23(0.78)$ & $2.31(0.74)$ & $2.13(0.86)$ & $2.26(0.71)$ \\
\hline
\end{tabular}


needing to be watched or kept company when awake. Dependence levels did not differ significantly across sites.

\section{DISCUSSION}

Achieving the goal of developing a predictor model for the course of Alzheimer disease is dependent on developing a cohort with mild disease severity that is of sufficient size and is representative of patients typically diagnosed with $\mathrm{pAD}$. The clinical diagnosis, staging of severity, and evaluation of potential components in the predictor model must be thorough and standardized, but optimally should use methods that could be reasonably applied in any clinical setting. The present report outlines our methodology toward meeting these goals. In order to achieve adequate statistical power, as well as to increase the generalizability of our findings, patients have been recruited from three different medical centers. These were representative of patients seen at medical centers specializing in $\mathrm{AD}$. Utilization of multiple study sites decreases the chance that a resulting predictor model would be applicable only to a particular site, with idiosyncratic diagnostic or evaluation procedures. Although the investigators at each site are adept at applying diagnostic criteria for $\mathrm{pAD}$, their available subject pool as well as their techniques for operationalizing diagnostic criteria differ to some degree. Also, referral issues at each site may differ because the $\mathrm{CU}$ site is primarily identified as a neurology department, $\mathrm{JH}$ as a psychiatry department, and MGH as a geriatric neurobehavioral center. Most importantly, our intended goal of developing a predictor model is dependent on identifying factors that account for differences in the rate of progression within the study cohort patients, so the cohort serves as its own control.

Differences in potential subject pools across sites may be partially responsible for the variability seen across sites in some measures. However, the compatibility of most indices across sites is notable. Of primary importance is that in all cases patients met standard criteria for $\mathrm{pAD}$ as well as study criteria designed to ensure that patients were of mild disease severity.

We were particularly concerned about the possibility that some study measures might be applied inconsistently. The most notable candidate in this regard is the assessment of extrapyramidal signs, since this requires a large degree of clinical judgment. A concurrent study performed at $\mathrm{CU}$ found good interrater reliability among three neurologists (Richards et al., 1991). However, these individuals were colleagues who trained and worked together. In order to maximize reliability of EPS ratings, several training and intersite reliability trials were conducted. These include visits of other sites' raters to CU as well as visits from CU to the two other sites. Formal estimates of interrater reliability for raters from the three sites was good. Despite these efforts, the frequency of EPS was higher at the JH and MGH sites than at CU. Some of this disparity is due to age differences across sites, particularly at MGH. It is notable that MGH reported higher frequencies of EPS whereas our reliability trials demonstrated a slight tendency for underrating signs at that site. Although some systematic overrating of EPS may exist, we are satisfied that on the whole, these differences in ratings represent actual differences in the frequency of EPS across sites. To the extent that intersite 
variability does exist, larger effect sizes would be required to find predictive utility for EPS.

Several new measures were developed specifically for the present study. The CUSPAD was developed as a brief, reliable assessment of psychopathology in pAD (Devanand et al., 1992). Although other scales for assessing psychopathology in $\mathrm{AD}$ exist, none fully met our study design requirements. We required a short instrument that could be administered by a lay interviewer with little formal training. To that end, the CUSPAD utilizes explicit questions that scan the spectrum of psychopathology that commonly occurs in $A D$ in order to determine the presence or absence of specific symptoms.

The dependence scale was also developed to meet the specific need for a severity measure or outcome that captures the amount of aid a patient is receiving, no matter what their living situation (Stern et al., 1992). A portion of patients included in the $\mathrm{MGH}$ cohort reside in a long-term care facility, but still meet all study inclusion and exclusion criteria. This suggests that nursing home entry per se is not a useful outcome for prediction, because even patients with mild disease may be institutionalized. The dependence scale investigates the actual amount of care a patient requires and is less liable to be influenced by sociological or financial factors that contribute to the decision to institutionalize a patient.

We chose to include patients with small subcortical lesions but without history or clinical evidence of stroke in our group with $\mathrm{pAD}$. To the extent that these lesions contribute to the presentation of dementia, some might feel that these patients would better be diagnosed with possible $\mathrm{AD}$ according to NINCDSADRDA criteria. However, the influence of the lesions on clinical presentation or disease course is an empirical issue that can be tested with the present cohort. Our preliminary evidence suggests that there is no difference between patients with and without these lesions at their initial presentation (Marder et al., 1992), but these findings must be confirmed and prospective data are required.

We also chose to include patients who were participating in experimental drug trials. Pragmatically, we felt that few patients with mild AD would consent to participate in the present study if it meant restricting access to therapeutic trials. Similarly, ethical considerations would dictate against such an exclusion criterion. From a design point of view, the potential effects of participation in these trials was considered a tolerable source of potential variation. Although some therapeutic trials have resulted in statistically significant improvements on criterion measures, the clinical significance of this improvement has been difficult to establish and is at best small. However, subjects' participation in these trials is carefully recorded so that potential drug effects on progression can be assessed or analyses can stratify by participation in these studies.

By standard measures such as the mMMSE, BDRS, duration of illness, and CDR, the cohort consisted of patients in the early stages of disease. For example, the average mMMSE score of the cohort is roughly equivalent to a score of 20 on the standard Mini-Mental. Still, EPS and behavioral disturbances were relatively common. This observation lends credence to their use as predictive signs: They are not simply signs of more advanced disease and occur early enough to be of potential value for predicting disease course. 
Acknowledgment: This study was supported by Federal grants AG07370, AG07232, AG08702, and the Charles S. Robertson Gift for Alzheimer's Disease.

\section{REFERENCES}

American Psychiatric Association. Diagnostic and statistical manual. third edition - revised, Washington, D.C.: A.P.A., 1987.

Bell K, Marder K, Sano M, Richards M, Miller L, Lafleche G, Mayeux R, Stern Y. Comparison of medical conditions and neurological examination in early Alzheimer's disease and normal elderly. Neurology 1992;42(suppl 3):199-200.

Blessed $G$, Tomlinson BE, Roth $M$. The association between quantitative measures of dementia and of senile changes in the cerebral grey matter of elderly subjects. Br J Psychiatry 1968;225:797811.

Davis PC, Gado M, Albert M, et al. CERAD protocol for assessing neuroimaging (MRI) findings in Alzheimer's disease. Neurology 1989;39(suppl 1):43.

Devanand DP, Miller L, Richards M, Marder K, Bell K, Mayeux R, Stern Y. The Columbia University Scale for Psychopathology in Alzheimer's Disease. Arch Neurol 1992;49:371-6.

Folstein MF, Folstein SE, McHugh PR. "Mini-Mental State"' a practical method for grading the cognitive state of patients for the clinician. J Psychiatr Res 1975;12:189-98.

Gurland B. Dependency in the elderly of New York City: policy and service implications of the US-UK Cross-national Geriatric Community Study. New York: Community Council of Greater New York, 1978.

Hachinski VC, lliff LD, Zhilka E, du Boulay GHD, McAllister V, Marshall J, Russell RWR, Symond L. Cerebral blood flow in dementia. Arch Neurol 1975;32:632-7.

Hamilton M. A rating scale for depression. J Neurol Neurosurg Psychiatry 1960;23:56-62.

Hughes CP, Berg L, Daniziger WL, Cohen LA, Martin RL. A new clinical scale for the staging of dementia. Br J Psychiatry 1982;140:566-72.

Marder K, Richards M, Bello J, Bell K, Sano M, Miller L, Lafleche G, Stern Y. Outcome and clinical correlates of Alzheimer's patients with "silent" stroke. Neurology 1992;42(suppl 3):142.

Mayeux R, Stern Y, Rosen J, Leventhal J. Depression, intellectual impairment and Parkinson's disease. Neurology 1981;31:645-50.

Mayeux R, Stern Y, Spanton S. Heterogeneity in dementia of the Alzheimer type: evidence of subgroups. Neurology 1985;35:453-61.

McKhann G, Drachman D, Folstein M, Katzman R, Price D, Stadlan EM. Clinical diagnosis of Alzheimer's disease: report of the NINCDS-ADRDA Work Group. Neurology 1984;34:939_ 44.

Richards M, Marder K, Bell K, Dooneief G, Mayeux R, Stern Y. Interrater reliability of extrapyramidal signs in a group assessed for dementia. Arch Neurol 1991;48:1147-9.

Sano M, Stern Y, Mayeux R, Hartman S, Devanand DP. A standardized technique for establishing the onset symptoms of probable Alzheimer's disease. J Clin Exp Neuropsychol 1987:9;65.

Stern MB. The clinical characteristics of Parkinson's disease and parkinsonian syndromes: diagnosis and assessment. In: Stern MB, Hurtig HI, eds. The Comprehensive Management of Parkinson's Disease. New York: PMA Publishing Corp. 1983:3-50.

Stern Y, Mayeux R, Sano M, Hauser WA, Bush T. Predictors of disease course in patients with probable Alzheimer's disease. Neurology 1987a;37:1649-53.

Stern Y, Sano M, Paulson J, Mayeux R. Modified Mini-Mental State Examination: validity and reliability. Neurology 37 (suppl 1);1987b:179.

Stern Y, Sano M, Miller L, Richards M, Marder K, Bell K, Bylsma F, Lafleche G. Assessing dependence, a unique feature of functional decline in Alzheimer's disease [Abstract]. Ann Neurol 1992;32:271.

Stern Y, Mayeux R, Hesdorfer D, Sano M. Measurement and prediction of functional change in Alzheimer's disease. Neurology 1990;40:8-14.

Wasson JH, Sox HC, Neff RK, Goldman L. Clinical prediction rules: applications and methodological standards. N Engl J Med 1985;313:793-9.

Wechsler D. Wechsler Adult Intelligence Scale. New York: Psychological Corporation, 1955.

Williams JB. A structured interview guide for the Hamilton Depression Rating Scale. Arch Gen Psychiatry 1988;45:742-7. 